\title{
An Experimental Comparison of the Geometry of Models Trained on Natural Language and Synthetic Data
}

\author{
Vincent Sippola ${ }^{\dagger}, *$, Robert E. Mercer ${ }^{\dagger}$ \\ $\dagger$ The University of Western Ontario
}

\begin{abstract}
Deep learning models have been used successfully to solve many Natural Language Processing problems, but less is known about the mechanisms that make them work. Unlike simpler models that can be understood mathematically, experimental comparisons and probing tasks are being used on deep neural net model attributes to try and determine how the syntactic and semantic information is being represented. In this work, we focus on models that produce contextual word vectors and sentence vectors. To determine how the information in these embeddings is being represented, we use a class of feed-forward neural networks to design a set of experiments that are run on the normal downstream tasks of two common Natural Language Inference benchmarks and one Sentiment Analysis benchmark, together with the classification tasks of a set of synthetic datasets that we have created. A quantitative analysis of these experiments shows that the behaviour of the models trained on the natural language benchmark data match those of the synthetic data in many cases. We suggest that this similar behaviour implies that the decomposition of semantic and syntactic data in the models trained on the language data are similar to the known structures of the synthetic data.
\end{abstract}

Keywords: Machine Learning, Natural Language Processing, Word Embeddings, Sentence Embeddings, Feed-forward Neural Networks

\section{Introduction}

With the rise of machine learning came the need to explain the behaviours of the models and explain the information that the model had learned. Mathematical methods have been used, but with deep and complex models, experimental methods are necessary to obtain such information. Various properties of a model can be examined: model parameters; sentence embeddings; Contextual Word Embeddings (CWEs); down-stream task performance; etc.

In this paper we propose a set of experiments that involve a class of feed-forward neural networks (FFNNs) that we call "Self-Enriching" layers, as they learn only from the information contained in the CWEs or sentences vectors, as opposed to enriching the embeddings with auxiliary information. This can be viewed as the complement to the work of Kovaleva, Romanov, Rogers, and Rumshisky [1], as we add FFNNs after layers that produce CWEs or sentence embeddings as opposed to disabling layers as they did.

We focus on variations of the BiLSTM architecture described by Conneau, Kiela, Schwenk, Barrault, and Bordes [2] to solve three downstream tasks on common benchmark sets. Additionally, we add a set of classification tasks using synthetic datasets at both the "word" and "sentence" level. The intent is to compare the behaviour of these tasks as the architectures are modified with Self-Enriching layers. We then use the similarity of these behaviours over all experiments to suggest that the underlying geometries share some similarities. Full results can be found in Sippola [3].

The paper is organized as follows: related work; the datasets used; the architectures; the experimental setup; the experimental results; and a conclusion and future work.

*vsippola@uwo.ca

This article is () 2021 by author(s) as listed above. The article is licensed under a Creative Commons Attribution (CC BY 4.0) International license (https://creativecommons.org/licenses/by/4.0/legalcode), except where otherwise indicated with respect to particular material included in the article. The article should be attributed to the author(s) identified above. 


\section{Related Work}

Previous work falls into two approaches: mathematical, which we describe first, and experimental, which is the methodology used in this paper. Levy and Goldberg [4] and Yang, Dai, Salakhutdinov, and Cohen [5] use a mathematical approach to examine the methods of Skip-gram with Negative Sampling (SGNS) and Neural Network Language Models (NNLM) respectively. They find that each method is implicitly its own type of matrix factorization. Ethayarajh [6] uses three different cosine "measures" to examine the geometry of the CWEs of BERT [7], GPT-2 [8], and ELMo [9].

Mimno and Thompson [10] use a mixed approach, varying the parameters of the SGNS algorithm and examining the space of vectors with the dot product. Press and Wolf [11] experimentally tie the weights of NNLM together and examine the baseline and tied CWEs. The SentEval toolkit from Conneau and Kiela [12] is designed for the standardized experimental comparison of models. Wieting and Kiela [13] use SentEval to compare randomly initialized models to models that were trained on linguistic data.

Mickus, Paperno, Constant, and Deemter [14] use pairs of sentences to measure the cohesion of BERT CWEs. Tang, Sennrich, and Nivre [15] use sentences with ambiguous nouns to find which parts of Transformer are solving Word Sense Disambiguation (WSD). Reif, Yuan, Wattenberg, Viegas, Coenen, Pearce, and Kim [16] use a classifier on BERT outputs to test where syntactic data is stored, and examine the CWE distributions to find WSD information. They suggest BERT spaces can be decomposed into distinct "subspaces". Kovaleva, Romanov, Rogers, and Rumshisky [1] examine the attention patterns of BERT models by disabling attention heads and layers during downstream task fine-tuning.

\section{Datasets}

Three publicly available datasets have been used: IMDb, SICK, and SNLI. The IMDB dataset [17] is designed for a Sentiment Analysis task [18]. 50K reviews are labelled either Positive or Negative; $25 \mathrm{~K}$ samples for training, and $25 \mathrm{~K}$ for testing. A random $2.5 \mathrm{~K}$ samples from the training set are used as a validation set. The SICK (10K examples) and SNLI (570K examples) datasets are corpora dealing with a Natural Language Inference (NLI) task [19]. The suggested training, validation, and testing splits are used.

Synthetic dataset 1 (SYNTH1) consists of 160 vectors of size 40 drawn from the normal distribution $\mathcal{N}(0,1)$ : 64 vectors for the training set, 32 for the validation set, and 64 for the testing set. For each set, the vectors are divided into 4 equally sized partitions, representing 4 classes, giving samples $\left(v_{i}, y_{i}\right): v_{i}$ is the vector; $y_{i}$ is the class label $1,2,3$, or 4 ; and vectors with label $y_{i}=j$ have the $j$ th quarter of the dimensions set to 0 , providing four highly overlapped clusters of vectors.

Specific vectors in class 1 are of the form $\sum_{10<i<40} \mathcal{N}(0,1) e_{i}$. To create the training examples for SYNTH1, the samples are paired into $\left(\left(v_{i}, v_{j}\right), y_{i, j}\right)_{1 \leq i, j \leq 64}$, where $y_{i_{j}}=1$ if $v_{i}=v_{j}, 0$ otherwise. The resulting 3600 examples, biased to label 0 , are rebalanced to give approximately 1800 examples with almost balanced labels. The validation and testing examples are created similarly, giving approximately 512 and 1800 examples, respectively.

To create SYNTH1n, two noising functions $M_{1}$ and $M_{2}$ are created as $40 \times 60$ dimensional random matrices from $\mathcal{N}(0,1)$. The examples are then transformed into $\left(\left(v_{i} \cdot M_{1}, v_{j}\right.\right.$. $\left.\left.M_{2}\right), y_{i, j}\right)$. Noising is meant to simulate different sentence embedding sources: different word embeddings, different encoding techniques, etc.

Synthetic dataset 2 (SYNTH2), created with the scikit-learn toolkit "make_classification" algorithm [20], provides 11,000 vectors for the two-class classification problem: 5000 training set, 1000 validation set, and 5000 testing set. Similar to SYNTH1, the samples for SYNTH2 are $\left(v_{i}, y_{i}\right)$, and two noising functions are created of size $20 \times 30$ giving SYNTH2n 
with samples $\left(\left(v_{i} \cdot M_{1}, v_{i} \cdot M_{2}\right), y_{i}\right)$. Two vector-perturbed datasets are created to simulate sequences of word embeddings. For SYNTH2s, 30 vectors are duplicated from each example in SYNTH2, 30 vectors sampled from $\mathcal{N}(0,0.1)$ are pairwise added to the first set which is then split into two sets of 15 vectors. The examples in SYNTH2s are the paired sequences of 15 vectors. SYNTH2ns is defined similarly using SYNTH2n.

\section{Model Descriptions}

Each experiment is done on pairs of sentences as this allows us to vary the way that the two sentence embeddings are combined before being fed to a classifier. Additionally, we use a class of feed-forward neural networks (FFNNs), which we call Self-Enriching layers, to modify parts of the architecture before the sentences are combined.

The baseline model chosen for the IMDb, SICK, and SNLI experiments is the InferSent BiLSTM architecture [2]. We choose max-pooling as our aggregation method to create a sentence representation from the BiLSTM CWEs, and modify the dimension of the BiLSTM to 300. The two layer classifier remains the same, with a hidden dimension of 512. For the synthetic datasets, each baseline architecture has a two layer classifier, with hidden dimension half the size of the combined vectors. For SYNTH2 the vector is repeated. The SYNTH2s and SYNTH2ns datasets additionally have two variations, one with a meanpooling and one with a max-pooling aggregation layer before sentence combination.

As we are always dealing with sentence pairs, we need to combine two sentence embeddings to feed to a classifier for our downstream task. Given two sentence embeddings $u$ and $v$, we use three combination methods: the naïve simple concatenation $(u, v)$; concatenation of the pairwise absolute value and the pairwise multiplication $(|u-v|, u * v)$, which is used by Le and Xie [21]; and concatenation of all three $(u, v,|u-v|, u * v)$, which is the standard method of combining vectors for many Natural Language Inference tasks [2, 19, 22].

Self-Enriching layers, which are used to modify the baseline architectures described above, can be described by a short 'Enriching code' that describes its structure. Here, enriching codes are of the form $M X_{1} \ldots X_{M} . M$ is the number of linear sublayers in the Self-Enriching layer (here, 1 or 2) and each $X_{i}$ describes the relative size of the input compared to the output in layer $i$ : e means 1:1, 1 means 1:2, and s means $2: 1$. To limit the number of experiments, we focus on only these 12 FFNNs. The activation function between two sublayers is ReLU, and in all sublayers the inputs and outputs are fully connected.

Self-Enriching layers are used in two ways. They are used in the IMDb, SICK, SNLI, SYNTH1, SYNTH1n, SYNTH2, and SYNTH2n datasets at the sentence level, treating the synthetic vectors as sentence embeddings. At the word embedding level, they are used in the SICK, SNLI, SYNTH2s and SYNTH2ns datasets before either the mean- or max-pooling layer. Each architecture is modified by at most one Self-Enriching layer per experiment.

\section{Experimental Setup and Results}

Each synthetic dataset is used as described in Section 3. FastText [23] vectors are used as input word embeddings for the SICK and SNLI datasets. Skip-gram and Continuous Bag of Words vectors are used for the IMDb dataset, giving us pairs of sentences.

We perform the following set of experiments, each with a baseline architecture and the hyperparameters appropriately chosen. We modify either the "word" or "sentence" vectors by a set of Self-Enriching layers as described in Section 4. Due to the long training times for SNLI and IMDb, we examine Self-Enriching with only one sublayer, and only at the sentence level for IMDb. For all of the other datasets we use the full set of Self-Enriching code combinations with one and two sublayers. 
Table 1. The average percentage of trials that performed better than the baseline for one- and two-sublayer Self-Enriching for each set of experiments. NL is the number of sublayers that the corresponding Self-Enriching layers have in the experiment sets. SYNTH datasets are labelled with the final characters of the SYNTH name. $\dagger$ represents max-pooling as the aggregation method. $\ddagger$ represents mean-pooling as the aggregation method. SICK and SNLI have Self-Enriching layers at the (S)entence level and the (W)ord level. IMDb has a Self-Enriching layer at the sentence level only.

\begin{tabular}{|c|c|c|c|c|c|c|c|c|c|c|c|c|c|c|}
\hline $\begin{array}{c}\text { Combination } \\
\text { Type }\end{array}$ & \multirow{2}{*}{ NL } & \multicolumn{10}{|c|}{ SYNTH } & \multicolumn{2}{c|}{ SICK } & \multicolumn{2}{|c|}{ SNLI } & \multirow{2}{*}{ IMDb } \\
\cline { 3 - 16 } & & 1 & $1 \mathrm{n}$ & 2 & $2 \mathrm{n}$ & $2 \mathrm{~s} \dagger$ & $2 \mathrm{~s} \ddagger$ & $2 \mathrm{~ns} \dagger$ & $2 \mathrm{~ns} \ddagger$ & $\mathrm{S}$ & $\mathrm{W}$ & $\mathrm{S}$ & $\mathrm{W}$ & \\
\hline \multirow{2}{*}{$\mathrm{u}, \mathrm{v}$} & 1 & 0 & 55 & 18 & 1 & 2 & 14 & 2 & 1 & 77 & 100 & 0 & 100 & - \\
& 2 & 0 & 70 & 37 & 16 & 24 & 48 & 7 & 1 & 100 & 100 & - & - & - \\
\hline \multirow{2}{*}{$|\mathrm{u}-\mathrm{v}|, \mathrm{u}^{*} \mathrm{v}$} & 1 & 0 & 65 & 99 & 100 & 100 & 100 & 89 & 76 & 0 & 0 & 0 & 100 & 100 \\
& 2 & 0 & 80 & 100 & 100 & 100 & 100 & 100 & 100 & 0 & 0 & - & - & - \\
\hline \multirow{2}{*}{$\mathrm{u}, \mathrm{v},|\mathrm{u}-\mathrm{v}|, \mathrm{u}^{*} \mathrm{v}$} & 1 & 0 & 43 & 54 & 70 & 26 & 30 & 80 & 62 & 0 & 63 & 0 & 45 & - \\
& 2 & 0 & 60 & 4 & 2 & 11 & 5 & 47 & 32 & 0 & 0 & - & - & - \\
\hline
\end{tabular}
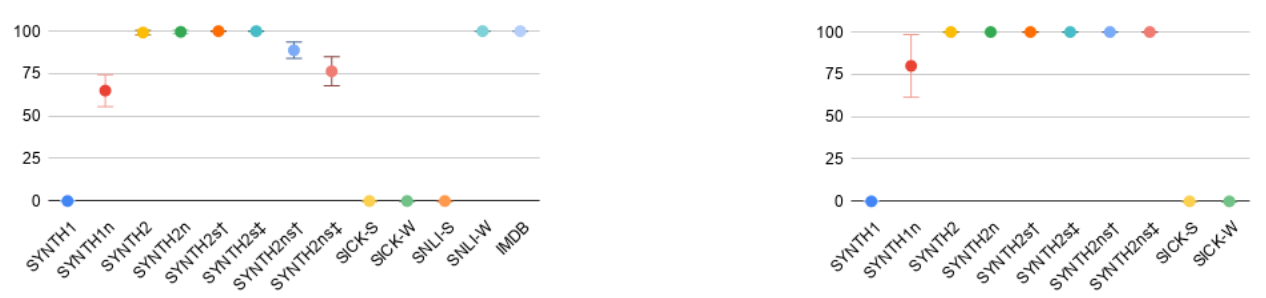

Figure 1. Visualization of the percentage of trials that performed better than the baseline for the $\left(|\mathrm{u}-\mathrm{v}|, \mathrm{u}^{*} \mathrm{v}\right)$ combination. Dots represent averages over all experiments and metrics with standard deviation. Left: One-sublayer averages Right: Two-sublayer averages.

To make sure we are getting a proper understanding of our models' behaviours, we run five trials of each experiment and track multiple metrics, an experiment being one architecture trained end-to-end on a downstream task. The length of this paper allows only a snapshot of the complete set of results of an in-depth study [3]. The discussion that follows sometimes refers to conclusions that are justified by that study.

We compare the relative increase or decrease in performance compared to the baseline on the downstream task, by examining the average of all experiments and metrics for one- and two-sublayers for each category and report these averages in Table 1 . Then, dividing the set $[0,100]$ into even quarters, we define two sets of experiments to be one-sublayer similar if their one-sublayer averages are in the same quarter. Two-sublayer similarity is defined in the same way.

Looking at the averages in Table 1 for the $(\mathrm{u}, \mathrm{v})$ combination type, SICK Sentence and SICK Word are similar for both one- and two-sublayers, with SNLI Word also one-sublayer similar. Besides SYNTH1n, all of the synthetic variants and SNLI Sentence are onesublayer similar. SYNTH1, SYNTH2n, SYNTH2s max-pooling, SYNTH2sn max-pooling, and SYNTH2sn mean-pooling are additionally two-sublayer similar. SYNTH1n is dissimilar to all other experiments, and the other two synthetic sets are two-sublayer similar.

For the $\left(|\mathrm{u}-\mathrm{v}|, \mathrm{u}^{*} \mathrm{v}\right)$ combination type, SICK Sentence, SICK Word, SNLI Sentence and SYNTH1 are all one-sublayer similar and two-sublayer similar, where possible. All SYNTH2 datasets are both one- and two-sublayer similar to each other, one-sublayer similar to SNLI word, and two-sublayer similar to SYNTH1n. Figure 1 visualizes the analysis for this combination.

For the $\left(\mathrm{u}, \mathrm{v},|\mathrm{u}-\mathrm{v}|, \mathrm{u}^{*} \mathrm{v}\right)$ combination type, SICK Sentence, SNLI Sentence, and SYNTH1 are one-sublayer similar. SYNTH1n, SYNTH2s max-pooling and mean-pooling, and SNLI 
word are one-sublayer similar as are SYNTH2, SYNTH2n, and SYNTH2ns mean-pooling. SYNTH1, SICK sentence and word, and all SYNTH2 datasets are two-sublayer similar except SYNTH2ns mean- and max-pooling which are two-sublayer similar to each other.

We note that the choice of combination type is important. With two exceptions, SYNTH1 and SNLI Sentence, no architecture shows consistent behaviour across all combination type. An in-depth study [3] has shown that simple concatenation of the embeddings shows consistently worse performance when compared to the corresponding baselines, but has the largest variety among the experiment sets, with most being worse than the baseline on average. For these reasons we do not compare results across combination types.

We note that SICK and SNLI with their normal combination type $\left(\mathrm{u}, \mathrm{v},|\mathrm{u}-\mathrm{v}|, \mathrm{u}^{*} \mathrm{v}\right)[2]$ behave very similarly to Synthetic Dataset 2 . These two NLI tasks, at the word level, are onesublayer similar, or very close to similar, with all of the SYNTH2 datasets, occupying mostly the middle ranges of 26-80. SICK being both one- and two-sublayer similar to SYNTH2 and SYNTH2n, and SNLI is similar to both SYNTH2s variants. This experimental evidence, to us, suggests that the geometries of these spaces are similar. Because the Synthetic datasets have been constructed of clusters of vectors representing different properties that are then combined, we hypothesize that the SICK and SNLI spaces have a similar geometry.

We note that the synthetic datasets agree when they are expected to. Knowing the construction of the synthetic datasets means we know when they should have similar behaviour. With the $\left(|\mathrm{u}-\mathrm{v}|, \mathrm{u}^{*} \mathrm{v}\right)$ combination type, all of the SYNTH2 variants are one- and two-sublayer similar. With the $\left(\mathrm{u}, \mathrm{v},|\mathrm{u}-\mathrm{v}|, \mathrm{u}^{*} \mathrm{v}\right)$ combination type, all of the SYNTH2 variants are in either the upper 50-75 range, or the lower 75-100 range, meaning they are either onesublayer similar or almost one-sublayer similar. The SYNTH2sn variants are two-sublayer similar to each other, and similarly the non-SYNTH2sn variants are two-sublayer similar to each other. With the $(\mathrm{u}, \mathrm{v})$ combination, all SYNTH2 variants are one-sublayer similar, but in this case there is a larger variation in the two-sublayer results, with only the SYNTH2sn variants being clearly two-sublayer similar. SYNTH1 is altogether different. It is left to the readers to confirm, but for each type of combination, a series of simple if statements could solve the classification problem with $100 \%$ accuracy. SYNTH1n is also different than the SYNTH2 variants as the original SYNTH1 clusters have lots of overlap, while the original SYNTH2 clusters do not. So we expected the observed contrast between the SYNTH1 results and the SYNTH2 results. Hence, when looking at one-sublayer similarity, we see results that clearly match our expectations, and when looking at two-sublayer, besides the minor variation in the $(\mathrm{u}, \mathrm{v})$ case, we get results that match our expectations. This analysis shows that our methodology is at least consistent when we know it should be.

\section{Conclusion and Future Work}

In this paper we have presented an experimental framework, using a restricted class of feed-forward neural networks, to compare compatible models. These experiments allow us to compare the behaviour of models trained on real language data and models trained on synthetic data to see if we can infer experimental similarity. We confirmed that in this framework the synthetic datasets behave in line with our expectations. We further observe that under this experimental framework the default NLI problems create word embeddings that are similar to our SYNTH2 dataset and its basic variations.

Going forward, as comparisons have been seen to be combination type dependant, it seems better to focus on one combination type or datasets that only use one sentence input but can still use the framework. Additionally, a larger and more diverse set of synthetic data should be used to get better baseline behaviours to compare against. Lastly, where possible, the architecture should be viewed with additional analyses. Some effort in this direction has already begun to provide a different view of the architectures [3]. 


\section{Acknowledgements}

This research was partially funded by The Natural Sciences and Engineering Research Council of Canada (NSERC) through a Discovery Grant to Robert E. Mercer. We also acknowledge the helpful comments provided by the reviewers.

\section{References}

[1] O. Kovaleva, A. Romanov, A. Rogers, and A. Rumshisky. "Revealing the dark secrets of BERT". In: Proc. of the 2019 Conf. on Emp. Meth. in Nat. Lang. Proc. and the 9th Int. J. Conf. on Nat. Lang. Proc. 2019, pp. 4365-4374.

[2] A. Conneau, D. Kiela, H. Schwenk, L. Barrault, and A. Bordes. "Supervised learning of universal sentence representations from Natural Language Inference data". In: arXiv preprint arXiv:1705.02364 (2017).

[3] V. Sippola. "Exploring explicit and implicit feature spaces in Natural Language Processing using self-enrichment and vector space analysis". Electronic Thesis and Dissertation Repository. 7471. MSc thesis. The University of Western Ontario, 2020.

[4] O. Levy and Y. Goldberg. "Neural word embedding as implicit matrix factorization". In: Proc. of the 27th Int. Conf. on Neural Inf. Proc. Systems - Vol. 2. 2014, pp. 21772185.

[5] Z. Yang, Z. Dai, R. Salakhutdinov, and W. W. Cohen. "Breaking the Softmax bottleneck: A high-rank RNN language model". In: arXiv preprint arXiv:1711.03953 (2017).

[6] K. Ethayarajh. "How contextual are contextualized word representations? Comparing the geometry of BERT, ELMo, and GPT-2 embeddings". In: Proc. of the 2019 Conf. on Empirical Methods in Nat. Lang. Proc. and the 9th Int. Joint Conf. on Nat. Lang. Proc. 2019, pp. 55-65.

[7] J. Devlin, M.-W. Chang, K. Lee, and K. Toutanova. "BERT: Pre-training of deep bidirectional transformers for language understanding". In: arXiv preprint arXiv:1810.04805 (2018).

[8] A. Radford, J. Wu, R. Child, D. Luan, D. Amodei, and I. Sutskever. "Language Models are Unsupervised Multitask Learners". In: $n / a$ (2018).

[9] M. Peters, M. Neumann, M. Iyyer, M. Gardner, C. Clark, K. Lee, and L. Zettlemoyer. "Deep contextualized word representations". In: Proc. of the 2018 Conf. of the N. Amer. Chap. of the ACL: Human Lang. Tech., Vol. 1 (Long Papers). 2018, pp. 2227-2237.

[10] D. Mimno and L. Thompson. "The strange geometry of skip-gram with negative sampling". In: Proc. of the 2017 Conf. on Empirical Methods in Nat. Lang. Proc. 2017, pp. 2873-2878.

[11] O. Press and L. Wolf. "Using the Output Embedding to Improve Language Models". In: Proceedings of the 15th Conference of the European Chapter of the Association for Computational Linguistics: Volume 2, Short Papers. 2017, pp. 157-163.

[12] A. Conneau and D. Kiela. "SentEval: An evaluation toolkit for universal sentence representations". In: arXiv preprint arXiv:1803.05449 (2018).

[13] J. Wieting and D. Kiela. "No training required: Exploring random encoders for sentence classification". In: arXiv preprint arXiv:1901.10444 (2019).

[14] T. Mickus, D. Paperno, M. Constant, and K. van Deemter. "What do you mean, BERT?" In: Proceedings of the Society for Computation in Linguistics 2020. 2020, pp. 279-290.

[15] G. Tang, R. Sennrich, and J. Nivre. "An analysis of attention mechanisms: The case of word sense disambiguation in neural machine translation". In: Proceedings of the Third Conference on Machine Translation: Research Papers. 2018, pp. 26-35.

[16] E. Reif, A. Yuan, M. Wattenberg, F. B. Viegas, A. Coenen, A. Pearce, and B. Kim. "Visualizing and measuring the geometry of BERT". In: Advances in Neural Information Processing Systems. Vol. 32. 2019, pp. 8594-8603.

[17] A. L. Maas, R. E. Daly, P. T. Pham, D. Huang, A. Y. Ng, and C. Potts. "Learning word vectors for sentiment analysis". In: Proc. of the 49th Annual Meeting of the Assoc. for Comp. Ling.: Human Language Technologies. 2011, pp. 142-150.

[18] B. Pang and L. Lee. "A Sentimental Education: Sentiment Analysis Using Subjectivity Summarization Based on Minimum Cuts". In: arXiv preprint cs/0409058 (2004).

[19] S. R. Bowman, G. Angeli, C. Potts, and C. D. Manning. "A large annotated corpus for learning natural language inference". In: Proc. of the 2015 Conf. on Empirical Methods in Natural Language Processing. 2015.

[20] F. Pedregosa, G. Varoquaux, A. Gramfort, V. Michel, B. Thirion, O. Grisel, M. Blondel, P. Prettenhofer, R. Weiss, V. Dubourg, J. Vanderplas, A. Passos, D. Cournapeau, M. Brucher, M. Perrot, and E. Duchesnay. "Scikit-learn: Machine Learning in Python". In: Journal of Machine Learning Research 12 (2011), pp. 2825-2830.

[21] L. Le and Y. Xie. "Deep embedding kernel". In: arXiv preprint arXiv:1804.05806 (2018). 
[22] M. Marelli, S. Menini, M. Baroni, L. Bentivogli, R. Bernardi, and R. Zamparelli. "A SICK cure for the evaluation of compositional distributional semantic models". In: Proc. of the Ninth Int. Conf. on Lang. Res. and Evaluation. 2014, pp. 216-223.

[23] T. Mikolov, E. Grave, P. Bojanowski, C. Puhrsch, and A. Joulin. "Advances in pre-training distributed word representations". In: Proc. of the International Conference on Language Resources and Evaluation. 2018, pp. 52-55. 